\title{
Prediction of nonlinear propagation of noise from a solid rocket motor
}

\author{
Michael B. Muhlestein, Kent L. Gee, Tracianne B. Neilsen, and Derek C. Thomas \\ Department of Physics and Astronomy, Brigham Young University, Provo, UT, 84602 \\ mimuhle@gmail.com,kentgee@byu.edu,tbn@byu.edu,dthomas@byu.edu
}

\begin{abstract}
:
Nonlinear acoustic wave propagation predictions (Generalized Burgers equation-based) of noise propagation are compared with measurements from a static, horizontally-fired solid rocket motor over a range of 76-1220 $\mathrm{m}$ during an $80 \mathrm{~s}$ burn time. The modeling suggests the nature of the geometric spreading between 76 and $305 \mathrm{~m}$ varies from cylindrical at low-frequencies to spherical at high frequencies. The predicted waveforms and high-frequency spectral slopes associated with significant shock content are in agreement with properties of the measured noise. At $1220 \mathrm{~m}$, the relatively simple nonlinear model again approximates the measured spectrum despite the complexities of the measurement environment and atmospheric propagation.
\end{abstract}

\section{Introduction}

Although the noise from launch vehicles is intense, characterization of nonlinear phenomena in rocket noise studies has been relatively limited. Morfey ${ }^{1}$ empirically modeled the high-frequency energy transfer caused by nonlinear wave steepening for multiple rocket launches. McInerny and Olcmen ${ }^{2}$ analyzed time domain measurements of two different launch vehicles at different distances and found significant evidence of shock propagation, even several kilometers away. Nevertheless, the only current launch vehicle noise prediction tool relies entirely on incoherent monopole distributions radiating linearly, regardless of rocket size or thrust, ${ }^{3}$ with no mention of possible nonlinear propagation effects. This is likely a limitation because the noise propagation from military and other highpower aircraft has been shown to be appreciably nonlinear, despite significantly lower thrust. ${ }^{4-6}$ For example, the average vacuum thrust produced by a four-segment reusable solid rocket motor from the Space Shuttle is $13 \mathrm{MN},{ }^{7}$ approximately 70 times the maximum thrust from the F-35 Joint Strike Fighter, which has been the subject of two recent nonlinear propagation studies. ${ }^{8,9}$ Shepherd et al. ${ }^{10}$ further showed that the high-amplitude noise radiated from a spherical source at rocket-like levels undergoes significant changes at the high and low ends of the spectrum as it propagates.

Despite evidence for nonlinear phenomena in rocket noise, the relative importance of these effects in modeling efforts requires further evaluation. Nonlinearity may have important implications because the accelerations due to acoustic shocks, which are purely nonlinear in nature, can exceed $2000 \mathrm{~m} / \mathrm{s}^{2}$ (200 G's), ${ }^{11}$ and excessively load structures. On the other hand, the noise produced by these larger rocket motors and engines is of lower frequency because of the larger nozzles and appears to be produced over a much larger turbulent source region than military jets, both of which could lessen the significance of the nonlinearity.

Modeling of nonlinearity in broadband noise propagation dates back to work by Pernet and Payne ${ }^{12}$ who examined anomalously low absorption of high-frequency energy in the spectrum for noise of sufficient intensity. Pestorius and Blackstock ${ }^{13}$ developed a time-waveform propagation model based on the generalized Burgers equation (GBE) $)^{14}$ and successfully modeled noise propagation, including shock formation and coalescence, in a long pipe. Additional arbitrary waveform modeling developments took place in the context of nonlinear sonic boom propagation, ${ }^{15-17}$ but much of the recent interest has been the noise propagation from modern high-performance tactical aircraft. Nonlinear F/A-18E noise propagation was calculated by Gee et al., ${ }^{18}$ Brouwer, ${ }^{19}$ and Saxena et al. ${ }^{20}$ using different GBE-based algorithms. A more comprehensive treatment of the noise radiated by the F-22A Raptor was carried out by Gee et al. ${ }^{4,5}$ and algorithm refinements were incorporated in a study of the noise propagation from the F-35AA Joint Strike Fighter. ${ }^{8}$ In these latter studies of F-22A and F-35AA noise, excellent agreement between nonlinear models and measurements were achieved at a maximum comparison distance of $305 \mathrm{~m}(1000 \mathrm{ft})$.

Although previous studies ${ }^{1,2}$ examined the nonlinear propagation of in-flight launch vehicles, this paper treats the propagation of noise from a static, horizontally fired solid rocket motor (SRM). Consequently, these measurements allow for 
greater temporal averaging, for spectral analysis, and locating of microphones along radials both near and far from the source, for propagation analysis. In this paper, the GBE algorithm used previously by Gee et al. ${ }^{8}$ has been employed to model nonlinear propagation from a solid rocket motor. The results show how the extended nature of the source affects the modeling of the nonlinearity, and demonstrates the persistence of shocks in rocket noise fields at much greater distances than examined previously for military jets.

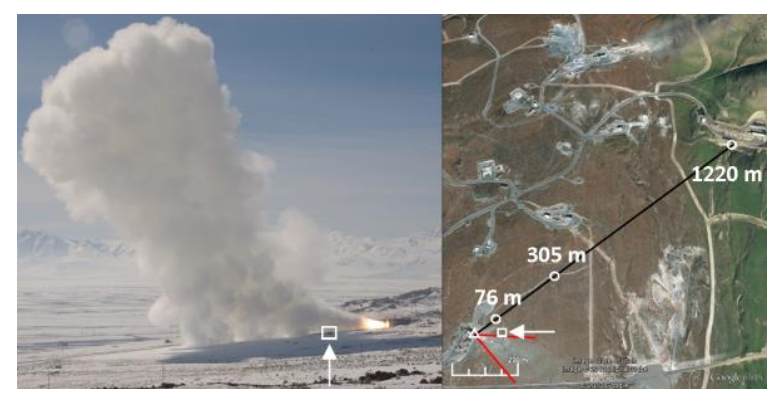

Figure 1. Picture of a GEM-60 SRM static firing and an annotated Google Earth ${ }^{\circledR}$ satellite image of the ATK test area. The triangle shows the location of the origin, and the circles show the locations of the three microphones along the radial $60^{\circ}$ relative to the downstream direction. A sense of scale is provided by rectangles in the picture and the map, both denoting the location of a large rock pile about $95 \mathrm{~m}$ from the rocket nozzle.

\section{Solid rocket motor static firing test meas- urement}

Noise measurements ${ }^{21}$ were made during a static firing of an ATK GEM-60 SRM, which is used with a Delta IV orbital launch vehicle and has 827 $\mathrm{kN}(186,000 \mathrm{lb})$ average thrust (see Fig. 1). The analyses in this paper are based on a data subset recorded using $6.35 \mathrm{~mm}$ GRAS 40BD pressure microphones at 76, 305, and $1220 \mathrm{~m}(250,1000$, and $4000 \mathrm{ft}$ ) from the chosen origin (about $10 \mathrm{~m}$ downstream of the nozzle) and along a $60^{\circ}$ radial relative to the plume axis. This angle likely approximates the peak directivity angle, based on vector intensity estimates ${ }^{21,22}$ and prior measurements of other solid rocket motors. ${ }^{3,7}$ The microphones were located 2-3 $\mathrm{m}$ above the ground, which was covered with about $15 \mathrm{~cm}$ of snow. The photograph in Fig. 1 was taken near the location of the $1220 \mathrm{~m}$ microphone, on top of a $45 \mathrm{~m}$ cliff and shows the sloping terrain surrounding the test site. The landscape and snow depth variability makes it difficult to quantify the effects of the terrain on the noise propagation. During the test, there was virtually no wind and the near-ground ambient pressure, temperature, and relative humid- ity were $87 \mathrm{kPa}, 3{ }^{\circ} \mathrm{C}$, and $60 \%$, respectively. Although the cloudless day and (anecdotally) warmer temperatures at the elevated observation location near the $1220 \mathrm{~m}$ microphone suggest downwardrefracting propagation conditions, large-scale temperature gradients were not quantified.

\section{Comparison of measured data with linear and nonlinear predictions}

The extended measurement time of the GEM-60 static firing allows ensemble-averaged comparison of measured spectra with predictions from both free-field linear and GBE-based nonlinear propagation models. First, however, a comparison of linearly and nonlinearly predicted waveforms, assuming spherical spreading, is presented to examine differences that point to the importance of nonlinearity in the noise propagation. Due to the complexities of the measurement terrain and of the source, a comparison of measured and predicted waveforms as was carried out in Ref. 4 is not intuitive, and are not presented. Waveform segments predicted at $305 \mathrm{~m}$ (based on the $76 \mathrm{~m}$ measured waveform) and $1220 \mathrm{~m}$ (based on the $305 \mathrm{~m}$ waveform) by the propagation models are shown in Fig. 2(a) and 2(b), respectively. In Fig. 2(a), the linearly predicted waveform exhibits significant smoothing of shocks by absorption, whereas the nonlinear prediction demonstrates propagation and coalescence of shock content. Figure 2(b) shows further the effects of atmospheric absorption in the linearly predicted $305 \mathrm{~m}$ waveform; between $305 \mathrm{~m}$ and $1220 \mathrm{~m}$, nearly all evidence of nonlinear steepening is eliminated. On the other hand, the nonlinear prediction still shows significant low-frequency shocks. In both cases, the differences between the linear and nonlinear model predictions imply that high-amplitude effects should be important to acoustic propagation extending to beyond $1 \mathrm{~km}$ from the GEM-60 SRM.

Although waveform steepening and shock propagation are observable in the time domain, their effects are best quantified in terms of ensemble-averaged spectra. In addition, the impact of linear phenomena neglected in the GBE model, e.g. multipath interference, are more naturally described in a spectral sense. Because the GEM-60 SRM was fired horizontally in a complex environment, ground reflections and scattering from nearby terrain are likely to manifest themselves in the measured spectra in the form of interference nulls and peaks. The measured spectra, shown as blue and black lines in Figs. 3 and 4, show that the 
measured spectra contain broad interference nulls around $180 \mathrm{~Hz}$ at $76 \mathrm{~m}, 125 \mathrm{~Hz}$ at $305 \mathrm{~m}$, and 60 $\mathrm{Hz}$ at $1220 \mathrm{~m}$. Although not exact, these nulls are reasonably predicted at the measurement locations from a monopole at the origin using the method of Embleton et $a .^{23}$ and an effective flow resistivity of 10-20 gcs rayls appropriate for snow-covered ground. However, given the drastic quantitative differences between a monopole and a rocket noise source distribution, these interference effects are noted, but not included, in the predictions.

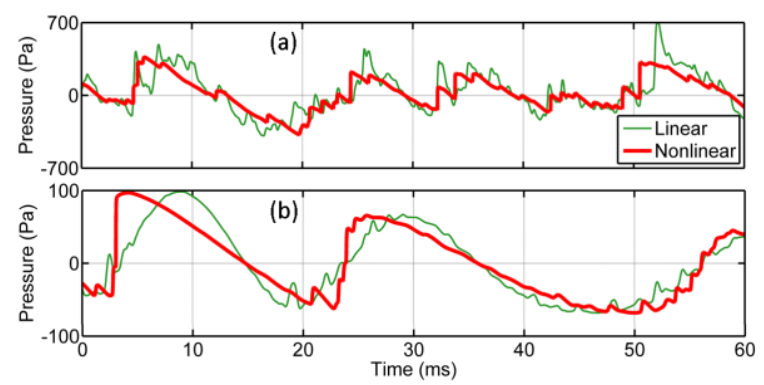

Figure 2. Linearly and nonlinearly predicted waveforms from the GEM-60 SRM firing. (a) $305 \mathrm{~m}$ predictions based on the 76-m measured waveform. (b) $1220 \mathrm{~m}$ predictions based on the $305 \mathrm{~m}$ measured waveform.

In making comparisons between measured and predicted spectra, one of the important considerations is the form of geometric spreading included in the GBE model. As mentioned previously, the spatial extent of the source causes the geometric spreading to be frequency and range dependent. For the GEM-60 SRM, Gee et al. ${ }^{21}$ found that the near-field OASPL 3-dB down points suggested the dominant source region extends about $50 \mathrm{~m}$. This large spatial extent implies the $76 \mathrm{~m}$ measurement location is subject to potential geometric near-field effects, including non-spherical spreading and a loss of spectral content from being upstream of low-frequency, directional radiation. Although the latter problem cannot be addressed with the onedimensional model, the former can be studied by comparing the results when cylindrical and spherical spreading are included over the 76-305 m propagation range.

The 76-305 m spectra predicted by cylindrical and spherical spreading for both linear and nonlinear propagation are shown in Figs. 3(a) and 3(b), respectively. Also shown are the input and measured spectra, along with overall sound pressure levels (OASPL) for all four curves, and guidelines showing a $f^{ \pm 2}$ dependence. These latter lines are provided because Gurbatov and Rudenko $^{24}$ determined that the power spectrum of broadband noise with well-developed weak shocks goes as $f^{-2}$ at high frequencies and as $f^{2}$ at low frequencies. Both the $76 \mathrm{~m}$ and $305 \mathrm{~m}$ measured spectra closely approximate these slopes at both high and low frequencies. A measured $f^{-2}$ highfrequency slope at $305 \mathrm{~m}$ is remarkable in and of itself. Prior measurements of the F-22A and F35AA aircraft showed excellent agreement between nonlinear predictions and measurements, but also that the high-frequency roll-off at $305 \mathrm{~m}$ was appreciably steeper than $f^{-2}$. This means that, for those cases, additional nonlinear waveform distortion had slowed relative to atmospheric losses, resulting a thickening of acoustic shocks such that they were no longer considered "weak" over the bandwidth of interest. ${ }^{25,24}$ However, in the case of this SRM, the shocks are sufficiently thin at $305 \mathrm{~m}$ to still possess this weak-shock slope out to 10 $\mathrm{kHz}$.
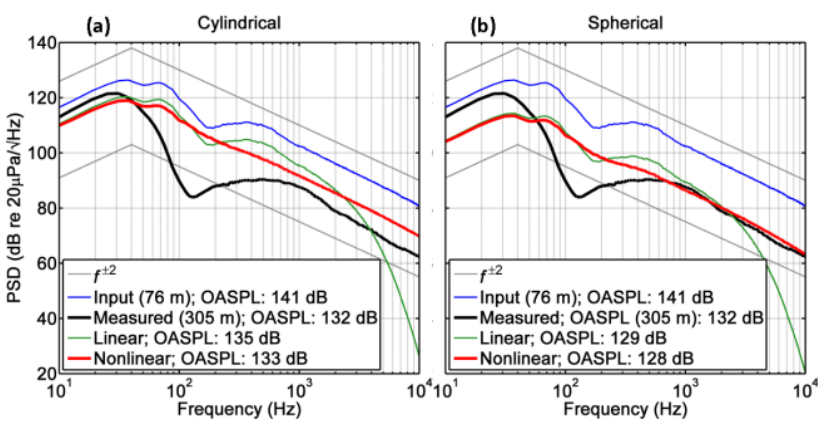

Figure 3. Measured spectra at 76 and $305 \mathrm{~m}$ from a GEM-60 SRM static firing, as well as the $76-305 \mathrm{~m}$ predictions (linear and nonlinear) assuming (a) cylindrical and (b) spherical geometric spreading.

In comparing the differences between the predictions for cylindrical and spherical spreading at low frequencies $(<30 \mathrm{~Hz})$, we see first that in both cases there is little difference between linear and nonlinear propagation. Second, we see that the measured low-frequency levels are much more closely approximated by cylindrical spreading (within 2-3 dB) than spherical spreading, where the difference between predicted and measured levels is approximately $8 \mathrm{~dB}$. In the $30-70 \mathrm{~Hz}$ peak-frequency region of both sets of predictions, there is a loss of energy due to nonlinearity, but more so in the case of cylindrical spreading because of the slower decrease in amplitude due to distance. This nonlinear energy transfer, primarily to higher frequencies to maintain shock-like profiles in the presence of absorption, results in a reduced OASPL for the nonlinear prediction relative to the linear prediction. 
Aside from the nonlinear net energy loss in the peak-frequency region, the difference between the linear and nonlinear predictions is most apparent at high frequencies, where atmospheric absorption has resulted in a $\sim 50 \mathrm{~dB}$ difference in level at $10 \mathrm{kHz}$. In addition, between $1-10 \mathrm{kHz}$, the spherical nonlinear model closely approximates the $305 \mathrm{~m}$ measured spectrum, with a $1.5 \mathrm{~dB}$ average difference, whereas the cylindrical spreading produces a similar shock-like slope but an overestimation of absolute level. Thus, the nonlinear model incorporating spherical spreading is more accurate in predicting the high-frequency noise propagation of rocket noise from $76 \mathrm{~m}$ to $305 \mathrm{~m}$.

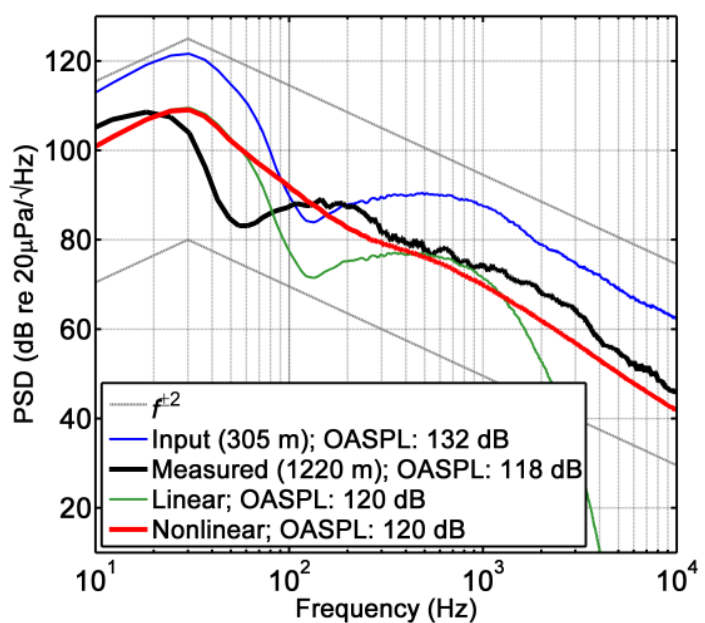

Figure 4. Measured spectra at 305 and $1220 \mathrm{~m}$ from a GEM-60 SRM static firing, as well as the 305-1220 m predictions assuming spherical spreading.

The combined results of Fig. 3(a) and 3(b), however, indicate that an input distance of $76 \mathrm{~m}$ (roughly 68 nozzle exit diameters) is not in the geometric far field at low frequencies, given the spatial extent and relative downstream origin of the noise source..$^{21,22,2}$ The propagation of the lowfrequency content is best modeled by cylindrical spreading in this case, but the one-dimensional GBE model may yield better or worse agreement for different choices of origin, propagation angle, or distance in the near field. On the other hand, at high-frequencies, the relative compactness of the dominant source region results in spherical spreading.

At a distance of $305 \mathrm{~m}$, the microphone is located sufficiently far from the plume to be considered in the far field. Thus, spherical spreading is incorporated into the modeling to make compar- isons over the $305-1220 \mathrm{~m}$ range. Figure 4 displays the $305 \mathrm{~m}$ and $1220 \mathrm{~m}$ measured spectra and the nonlinear and linear predictions over that range, using the $305 \mathrm{~m}$ waveform as input. Over this range, the predicted atmospheric absorption ${ }^{26}$ at 10 $\mathrm{kHz}$ is an astounding $194 \mathrm{~dB}$, such that the measured $46 \mathrm{~dB}$ level is significant evidence of nonlinear propagation by itself! The comparisons of the modeled spectra show very little difference in the peak-frequency region but extreme differences at high frequencies. The linear model indicates that no energy should be measurable above about 4 $\mathrm{kHz}$, yet the nonlinear model closely approximates the measurement at both low and high frequencies to within 3-5 $\mathrm{dB}$ at all frequencies outside the interference null region. The agreement provided by the nonlinear model is quite good considering the long range implementation of a free-field GBE model, the uncertainties present in real atmospheric propagation, including the possible downward refracting atmosphere near the ground. In addition, above about $1.5-2 \mathrm{kHz}$, the slopes of the measured and modeled spectra have begun to roll off more quickly than $f^{-2}$, suggesting thickening of the propagating shock fronts at $1220 \mathrm{~m}$.

\section{Conclusion}

This paper has described application of a generalized Burgers equation model to rocket noise propagation. The static, horizontal firing of a solid rocket motor resulted in a unique measurement situation, which allowed measurements to be taken at multiple locations along a single radial and the possibility of significant ensemble averaging. Both the measured and nonlinearly predicted waveforms suggest weak shocks $305 \mathrm{~m}$ from the origin, indicating more significant nonlinear propagation that previous military jet experiments. The propagation out to $1220 \mathrm{~m}$ is also fairly closely modeled via the GBE, suggesting ongoing nonlinear propagation out to those distances. In addition, the need for cylindrical spreading to more closely model the low-frequency propagation between 76$305 \mathrm{~m}$ speaks to the large extent of the aeroacoustic source region.

In some sense, it is remarkable that despite the free-field environment and neutral atmosphere assumptions, the GBE model is able to approximate the measured propagation in a relatively complex measurement environment. Outside the geometric near field, where the type of geometric spreading and choice of propagation radial is of concern, the principal difficulty is incorporating a 
correction for the significant ground interference null caused by the soft ground in this case. Future efforts may involve accounting for the effects of the ground in the model or further application to measurements made at the test area during different times of the year. However, despite the limitations of the current study, the ensemble-averaged nature of the nonlinearly modeled and measured spectra, and their relative agreement, clearly show the need to consider acoustic nonlinearities in spectral predictions of solid rocket motor noise propagation.

\section{Acknowledgements}

The authors are grateful to Kevin Rees and Roy Norris of ATK Test Services and for funding from NASA under a previous STTR grant.

\section{References}

${ }^{1}$ C. L. Morfey, "Aperiodic signal propagation at finite amplitudes: some practical applications," in Proceedings of the 10th International Symposium on Nonlinear Acoustics, Kobe, Japan (1984).

${ }^{2}$ S. A. McInerny and S. M. Olcmen, "High-intensity rocket noise: Nonlinear propagation, atmospheric absorption, and characterization," J. Acoust. Soc. Am. 117(2), 578-591 (2005).

${ }^{3} \mathrm{~K}$. M. Eldred, "Acoustic loads generated by the propulsion system," NASA SP-8072 (1971).

${ }^{4}$ K. L. Gee, V. W. Sparrow, M. M. James, J. M.

Downing, C. M. Hobbs, T. B. Gabrielson, and A. A. Atchley, "The role of nonlinear effects in the propagation of noise from high-power jet aircraft," J. Acoust. Soc. Am. 123(6), 4082-4093 (2008).

${ }^{5}$ K. L. Gee, V. W. Sparrow, M. M. James, J. M. Downing, C. M. Hobbs, T. B. Gabrielson, and A. A. Atchley, "Measurement and Prediction of Noise Propagation from a High-Power Jet Aircraft," AIAA J. 45(12), 3003-3006 (2007).

${ }^{6}$ C. L. Morfey and G. P. Howell, "Nonlinear Propagation of Aircraft Noise in the Atmosphere," AIAA J. 19(8), 986-992 (1981).

${ }^{7}$ R. J. Kenny, C. Hobbs, K. Plotkin, and D. Pilkey, "Measurement and characterization of space shuttle solid rocket motor plume acoustics," AIAA Paper No. 2009-3161 (2009).

${ }^{8}$ K. L. Gee, J. M. Downing, M. M. James, R. C. McKinley, R. L. McKinley, T. B. Neilsen, and A. T. Wall, "Nonlinear Evolution of Noise from a Military Jet Aircraft during Ground Run-up," AIAA Paper No. 2012-2258 (2012).

${ }^{9}$ K. L. Gee, T. B. Neilsen, J. M. Downing, M. M. James, R. L. McKinley, and R. C. McKinley, "Nearfield shock formation in noise propagation from a high-power jet aircraft," J. Acoust. Soc. Am. 133(2), EL88-EL93 (2013).

${ }^{10}$ M. M. James and K. L. Gee, "Advanced acoustic measurement system for rocket noise source characterization," in Internoise 2012 (New York City, 2012).

${ }^{11}$ M. R. Shepherd, K. L. Gee, and M. S. Wochner, "Short-range shock formation and coalescence in numerical simulation of broadband noise propagation," J. Acoust. Soc. Am. 126(6), 2886-2893 (2009).

${ }^{12}$ D. F. Pernet and R. C. Payne, "Non-linear propagation of signals in air," J. Sound Vib. 17(3), 383-396 (1971).

${ }^{13}$ F. M. Pestorius and D. T. Blackstock, "Propagation of finite-amplitude noise," in Finite-amplitude wave effects in fluids, 24-29 (Copenhagen, 1973) .

${ }^{14}$ D. T. Blackstock, M. F. Hamilton, and A. D. Pierce, "Progressive Waves in Lossless and Lossy Fluids," in Nonlinear Acoustics, M. F. Hamilton and D. T. Blackstock (Academic Press, 1998) Ch. 13.

${ }^{15}$ R. O. Cleveland, J. P. Chambers, H. E. Bass, R. Raspet, D. T. Blackstock, and M. F. Hamilton, "Comparison of computer codes for the propagation of sonic boom wavefomrs through isothermal atmospheres," J. Acoust. Soc. Am. 100(5), 30173027 (1996).

${ }^{16}$ L. D. Robinson, Sonic boom propagation through an inhomogeneous, windy atmosphere, Ph.D. dissertation, Dept. of Mechanical Engineering, The University of Texas at Austin, 1991.

${ }^{17}$ H. E. Bass, R. Raspet, J. P. Chambers, and M. Kelly, "Modification of sonic boom wave forms during propagation from the source to the ground," J. Acoust. Soc. Am. 111(1), 481-486 (2002).

${ }^{18}$ K. L. Gee, V. W. Sparrow, T. B. Gabrielson, and A. A. Atchley, "Nonlinear modeling of F/A-18E noise propagation," AIAA Paper No. 2005-3089 (2005).

${ }^{19}$ H. H. Brouwer, "Numerical Simulation of Nonlinear Jet Noise Propagation," AIAA Paper No. 2005-3088 (2005).

${ }^{20}$ S. Saxena, P. J. Morris, and K. Viswanathan, "Algorithm for the Nonlinear Propagation of Broadband Jet Noise," AIAA J. 47(1), 186-194 (2009).

${ }^{21}$ K. L. Gee, J. H. Giraud, J. D. Blotter, and S. D. Sommerfeldt, "Energy-Based Acoustical Measurements of Rocket Noise," AIAA Paper No. 2009-3165 (2009).

${ }^{22}$ K. L. Gee, J. H. Giraud, J. D. Blotter, and S. D. Sommerfeldt, "Near-field vector intensity measurements of a small solid rocket motor," J. Acoust. Soc. Am. 128(2), EL69-EL74 (2010).

${ }^{23}$ T. F. W. Embleton, J. E. Piercy, and G. A. Daigle, "Effective flow resistivity of ground surfaces 
determined by acoustical measurements," J. Acoust. Soc. Am. 74(4), 1239-1244 (1983).

${ }^{24}$ S. N. Gurbatov and O. V. Rudenko, "Statistical Phenomena," in Nonlinear Acoustics, M. F. Hamilton and D. T. Blackstock (Academic Press, 1998) Ch. 13. ${ }^{25}$ A. D. Pierce, Acoustics (Acoustical Society of America, New York, 1989).

${ }^{26}$ H. E. Bass, L. C. Sutherland, A. J. Zuckerwar, D. T. Blackstock, and D. M. Hester, "Atmospherica absorption of sound: Further developments," J. Acoust. Soc. Am. 97(1), 680-683 (1995), and "erratum: Atmospherica absorption of sound: Further developments," J. Acoust. Soc. Am. 99(2), 1259 (1996).Bass et al. Atmospheric absorption further developments and erratum. 